\title{
Communication Patterns in VANETs
}

\author{
Elmar Schoch*, Frank Kargl*, Tim Leinmüller ${ }^{+}$, Michael Weber* \\ ${ }^{*}$ Ulm University, Institute of Media Informatics, \\ \{elmar.schoch|frank.kargl|michael.weber\}@uni-ulm.de \\ ${ }^{+}$DENSO AUTOMOTIVE Deutschland GmbH, \\ t.leinmueller@denso-auto.de
}

\begin{abstract}
Vehicular networks are a very promising technology to increase traffic safety and efficiency and to enable numerous other applications in the domain of vehicular communication. Proposed applications for VANETs have very diverse properties and often require non-standard communication protocols. Moreover, the dynamics of the network due to vehicle movements further complicates the design of an appropriate, comprehensive communication system. In this work, we collect and categorize envisioned applications from various sources and classify the unique network characteristics of vehicular networks. Based on this analysis, we propose five distinct communication patterns that form the basis of almost all VANET applications. Both the analysis and the communication patterns shall deepen the understanding of VANETs and simplify further development of VANET communication systems.
\end{abstract}

Index Terms-Vehicular ad hoc networks (VANETs), applications, communication patterns, network characteristics

\section{INTRODUCTION}

Up to now, a number of research projects have been carried out to investigate the vision of communicating vehicles. In projects like FleetNet ${ }^{1}$ or $\mathrm{CarTALK}^{2}$, researchers designed protocols, algorithms and systems to test the general feasibility of wireless communication between vehicles. As the first generation projects both in the U.S. and Europe have finished their work some time ago, vehicular networks are under way of being both extended and consolidated at the same time. In a number of more recent research projects like Network-onWheels ${ }^{3}$ or the European projects SafeSpot ${ }^{4}$, CVIS $^{5}$, Coopers $^{6}$ both automotive industry and academia are addressing the topic and approach the vision in a less experimental way than previous initiatives. This intention is also corroborated by the fact that new consortia like the $\mathrm{C} 2 \mathrm{C}-\mathrm{CC}^{7}$ or the $\mathrm{VII}^{8}$ started the process to standardize systems and protocols. For instance, as a basis for the wireless communication, the U.S. FCC has allocated $75 \mathrm{MHz}$ of bandwidth for DSRC (Dedicated Short Range Communication) [1], which is now used by the emerging IEEE 802.11p standard.

Starting with the idea of making driving safer by intervehicle communication, the concept of vehicular networks or vehicular ad hoc networks (VANETs) has been extended to a large collection of various applications, which can profit

\footnotetext{
${ }^{1}$ http://www.et2.tu-harburg.de/fleetnet/ - FleetNet project

${ }^{2} \mathrm{http}: / /$ www.cartalk2000.net/ - CarTALK project

${ }^{3}$ http://www.network-on-wheels.de/ - NOW project

${ }^{4}$ http://www.safespot-eu.org/ - SafeSpot project

${ }^{5} \mathrm{http}: / /$ www.cvisproject.org/ - CVIS project

${ }^{6} \mathrm{http} / / / \mathrm{www}$. coopers-ip.eu - Coopers project

${ }^{7}$ http://www.car-to-car.org/ - Car-to-Car Communication Consortium

${ }^{8} \mathrm{http}: / /$ www.vehicle-infrastructure.org/ - Vehicle Infrastructure Integration
}

from wireless communication between vehicles. Nowadays, vehicles are not only envisioned to communicate between each other, but also to get information from and send data to infrastructural units. These stationary parts of the vehicular network range from traffic lights and dynamic traffic signs to access points at home, at gas stations or elsewhere. In addition, although active safety applications still represent the central idea, also traffic efficiency applications as well as entertainment and business applications have been proposed. In summary, the diverging requirements of all these applications make the design of a comprehensive communication system a very complex topic.

Due to this complexity and diversity of vehicular networks, we consider it helpful for the further development to analyze and structure proposals made in different projects so far. We therefore start with a comprehensive collection of envisioned applications in Section II Based on a broad overview of future applications and their requirements we then address network characteristics, as the communication system constitutes the central element of most applications. A lot of previous work exists on communication in VANETs, however, this work is very diverse and not well categorized yet. We will not present the different approaches individually, but instead distill and discuss five design patterns termed "VANET communication patterns" that form the basis of virtually all VANET applications envisioned so far. These patterns can serve both as a reference when reviewing existing work on VANET communication and also as a point of orientation for new approaches.

\section{ApPLICATIONS}

Applications based on vehicular communication range from simple exchange of vehicle status data to highly complex, large-scale traffic management including infrastructure integration. As a start to analyze applications, this section gives an overview on envisioned application categories for vehicular networks. Although exact operation details are not yet standardized for most applications and in spite that such a collection can never be completely finished, the overview delivers basic mechanisms, components and constraints involved in the system. This provides an initial understanding on the properties of VANET communication and will lead over to a more detailed analysis of network characteristics in the next section.

The applications presented in Table I are compiled from several sources. A large collection of applications was gathered in a report [2] by the Vehicle Safety Communications (VSC) 


\begin{tabular}{|c|c|c|}
\hline & Situation/Purpose & Application Examples \\
\hline \multirow[t]{5}{*}{ I. Active Safety } & 1. Dangerous road features & $\begin{array}{l}\text { 1. Curve speed warning, 2. Low bridge warning, 3. Warning about violated } \\
\text { traffic lights or stop signals }\end{array}$ \\
\hline & 2. Abnormal traffic and road conditions & $\begin{array}{l}\text { 1. Vehicle-based road condition warning, } 2 \text {. Infrastructure-based road condition } \\
\text { warning, 3. Visibility enhancer, 4. Work zone warning }\end{array}$ \\
\hline & 3. Danger of collision & $\begin{array}{l}\text { 1. Blind spot warning, } 2 \text {. Lane change warning, } 3 \text {. Intersection collision warning, } \\
\text { 4. Forward/Rear collision warning, } 5 \text {. Emergency electronic brake lights, } 6 \text {. Rail } \\
\text { collision warning, } 7 \text {. Warning about pedestrians crossing }\end{array}$ \\
\hline & 4. Crash imminent & 1. Pre-crash sensing \\
\hline & 5. Incident occurred & 1. Post-crash warning, 2. Breakdown warning, 3. SOS service \\
\hline \multirow[t]{2}{*}{ II. Public Service } & 1. Emergency response & $\begin{array}{l}\text { 1. Approaching emergency vehicle warning, 2. Emergency vehicle signal } \\
\text { preemption, 3. Emergency vehicle at scene warning }\end{array}$ \\
\hline & 2. Support for authorities & $\begin{array}{l}\text { 1. Electronic license plate, 2. Electronic drivers license, 3. Vehicle safety } \\
\text { inspection, 4. Stolen vehicles tracking }\end{array}$ \\
\hline \multirow[t]{2}{*}{ III. Improved driving } & 1. Enhanced Driving & $\begin{array}{l}\text { 1. Highway merge assistant, } 2 \text {. Left turn assistant, 3. Cooperative adaptive } \\
\text { cruise control, } 4 \text {. Cooperative glare reduction, } 5 \text {. In-vehicle signage, } 6 \text {. Adaptive } \\
\text { drivetrain management }\end{array}$ \\
\hline & 2. Traffic Efficiency & $\begin{array}{l}\text { 1. Notification of crash or road surface conditions to a traffic operation center, } \\
\text { 2. Intelligent traffic flow control, 3. Enhanced route guidance and navigation, } \\
\text { 4. Map download/update, 5. Parking spot locator service }\end{array}$ \\
\hline \multirow[t]{4}{*}{ IV. Business/Entertainment } & 1. Vehicle Maintenance & $\begin{array}{l}\text { 1. Wireless diagnostics, 2. Software update/flashing, 3. Safety recall notice, } 4 . \\
\text { Just-in-time repair notification }\end{array}$ \\
\hline & 2. Mobile Services & $\begin{array}{l}\text { 1. Internet service provisioning, 2. Instant Messaging, 3. Point-of-interest } \\
\text { notification }\end{array}$ \\
\hline & 3. Enterprise solutions & $\begin{array}{l}\text { 1. Fleet management, 2. Rental car processing, 3. Area access control, } 4 . \\
\text { Hazardous material cargo tracking }\end{array}$ \\
\hline & 4. E-Payment & 1. Toll collection, 2. Parking payment, 3. Gas payment \\
\hline
\end{tabular}

TABLE I

OVERVIEW ON APPLICATIONS FOR VANETS

project. Concentrating on active-safety, Dötzer et al. have categorized a number of applications in [3]. In the FleetNet project, a categorization of applications was presented in |4|, which also has some similarities to our classification, but is less detailed. A deeper description of virtual warning sign applications is given by Maihöfer et al. in [5]. Additionally, most publications on VANETs also contain examples of applications. The chosen classification scheme groups applications by their purpose, which leads to groups of logically similar applications.

\section{A. Active Safety}

Active safety applications are considered as the typical and most desirable group of applications for VANETs with direct impact on road safety. The basic intention is to make driving safer by communication which can mean that drivers are warned about a dangerous situation or even that the vehicle can try to avoid an accident or to react appropriately, if an accident cannot be avoided any more.

In Table I, we categorize active safety applications according to the danger level, which can be seen as a compilation of criteria elaborated in [3]. Dangerous road features like curves are static and thus foreseeable, thus danger is low. Abnormal traffic and road conditions are still almost static, but have a dynamic notion, i.e. differ from the expectation of drivers that regularly pass the event location. In these cases, danger is elevated. Danger is high when applications try to prevent collisions, e.g. if a vehicle brakes heavily in dense traffic. If this does not help any more, i.e. in case of imminent danger when a collision cannot be avoided any more, precrash-sensing will prepare the vehicle in order to minimize the impact of the impending crash, e.g. by closing windows or raising dampers. Finally, when danger turned to an incident, it is important to warn approaching vehicles or call for help.

\section{B. Public Service}

Vehicular networks are also intended to support the work of public service like police or emergency recovery units. Prominent examples of this category are the support of emergency vehicles by virtual sirens or signal preemption capabilities. Using these applications, emergency vehicles may be able to reach their destination much faster than today. In addition, traffic surveillance could be simplified by applications like an electronic license plate. However, such an application must not be abused by anyone, which clearly underlines security requirements and the need for a discussion of legal aspects of vehicular communication.

\section{Improved Driving}

This category contains applications that try to improve or to simplify driving by means of communication. The idea comprises microscopic scenarios in the immediate surrounding of a vehicle as well as macroscopic optimization of traffic efficiency. In the first case, helper applications are intended to assist the driver in standard traffic situations like when entering a motorway and merging into the flowing traffic or the cooperative reduction of glare due to upper beam headlights. In the second case, traffic efficiency in a greater area is targeted. This can mean that an accident warning is disseminated in a larger area to inform vehicles about the potential obstacle so that drivers can take a different route. Another service is the dissemination of parking information or even the reservation of a parking space. 


\section{Mobile Business and Entertainment}

A large block of applications can be embraced under the terms business and entertainment. Here, the focus is on delivering services to customers, automation of vehiclerelated tasks or payment applications, like download of music, fleet management, simpler vehicle maintenance or payment of parking or road usage.

\section{Network Characteristics}

Besides the application requirements another major set of constraints to the development of applications, respective message dissemination methods and security mechanisms is given by the network characteristics, which make VANETs a very distinct category of ad hoc networks. In this section, we categorize these characteristics and elaborate on consequences for development.

\section{A. Node Velocity}

One of the most important aspects of mobility in VANETs is the potential node velocity. Nodes either denote vehicles or road side units (RSUs) in this case. Node velocity may range from zero for stationary RSUs or when vehicles are stuck in a traffic jam to over 200 kilometers per hour on highways.

In particular, these two extremes each pose a special challenge to the communication system. In case of very high node velocities, the mutual wireless communication window is very short due to a relatively small transmission range of several hundred meters. For example, if two cars driving in opposite directions with $90 \mathrm{~km} / \mathrm{h}$ each, and if we assume a theoretical wireless transmission range of $300 \mathrm{~m}$, communication is only possible for 12 seconds. Moreover, the transceivers have to cope with physical phenomena like the Doppler effect. Because the link layer cannot predict when a connection will be disrupted, link failures will occur frequently. For routing or multi hop message dissemination these short encounters between vehicles and general movement lead to a highly unstable topology, rendering topology-based routing practically useless. In their review of challenges of inter-vehicle communication [6], Blum et al. show by means of simulations, that routes discovered by topology-based routing protocols get invalid even before they are fully established. For applications, high node velocities have the effect that e.g. context awareness gets difficult because the immediate context changes very fast.

In the other extreme, with almost no mobility, the network topology is much more stable. However, slow movements in the vehicular domain usually also mean a very high vehicle density, which results in high interference, medium access problems, etc. For such reasons, very scalable communication solutions are required.

\section{B. Movement Patterns}

Vehicles do not move around arbitrarily, but use predefined roads, usually in two directions. Unpredictable changes in the direction of vehicles usually only occur at intersections of roads.
We distinguish three types of roads:

\section{- Dense city road network}

Inside cities, the road density is relatively high. There are lots of smaller roads, but also bigger, arterial roads. Many intersections cut road segments into small pieces. Often, buildings right beside the roads limit wireless communication.

\section{- Rural roads}

These roads usually have much larger segments, which means that intersections are more rare than in cities. Traffic conditions often do not allow the formation of a connected network, because too few vehicles are on the road. The overall direction of rural roads changes more frequently than the direction of highways.

\section{- Highways}

Highways typically form a multi-lane road, which has very large segments and well-defined exits and on-ramps. Movements are quasi one-dimensional and highways usually keep their direction towards another city etc.

These movement scenarios pose special challenges particularly for the routing. In contrast to cities where the traffic is very unordered, vehicles on a highway form the other extreme, because almost only one dimension is left. This leaves virtually no margin for alternate routes, like Blum et al. also state in their work $|6|$. In their simulation of 9.2 miles of a highway, they also encountered frequent fragmentation and a link lifetime of only about 1 minute even when driving in the same direction (assuming $500 \mathrm{ft}$ radio range).

\section{Node Density}

Apart from speed and movement pattern, node density is the third key property of vehicular mobility. Like already introduced shortly, it is not hard to imagine that the number of other vehicles in mutual radio range may vary from zero to dozens or even hundreds. If we assume a traffic jam on a highway with 4 lanes, one vehicle at every 20 meters and a radio range of $300 \mathrm{~m}$, every node theoretically has 120 vehicles in his transmission range.

In case of very low density, immediate message forwarding gets impossible. In this case, more sophisticated information dissemination is necessary, which can store and forward selected information, when vehicles encounter each other. In this case, the same message may be repeated by the same vehicle multiple times. In high density situations, the opposite must be achieved. Here, a message should be repeated only by selected nodes, because otherwise this may lead to an overloaded channel.

In addition, node density is not only correlated to the type of road, but also to time. In the daytime, the density on highways or in cities is high enough for immediate forwarding, as long as the routing can deal with fragmentation. However, during the night, few vehicles are around on these kind of roads, too.

\section{Node Heterogeneity}

Among the nodes participating in the envisioned applications, we find numerous different kinds and types. A basic 
distinction can be made between vehicles and infrastructural units, often called road side units (RSU). Vehicles can be further categorized in private vehicles, authority vehicles, road construction and maintenance vehicles and so on. Certainly, not all applications will be installed in all vehicles, e.g. only a emergency vehicle should be able to issue warnings about its approach. The situation is similar for RSUs. Depending on the capabilities of the units, infrastructural nodes may simply emit data to the network or have complete ad hoc functionality and thus may be used for forwarding like other vehicles. Moreover, infrastructural nodes may provide access to background networks, e.g. to inform a traffic operation center about road conditions. In contrast to vehicles, RSUs have widely different capabilities. Regarding applications, they do not possess the same sensors like a vehicle and they do not process messages for presentation to the driver or for taking actions of the vehicle. Also unlike vehicles, these nodes are stationary and do not have a relation to persons or companies, so RSUs do not need to protect their privacy.

\section{Communication Patterns}

As shown, potential applications for VANETs and network characteristics diverge largely. Moreover, the operation of applications is usually not detailed yet, i.e. it is open how data is collected, communicated and evaluated to implement the application. However, discussions of various applications in different projects show that essentially communication in virtually all applications can be grouped into a small set of types. These recurring patterns with multiple, similar characteristics form an generic base for the design of VANET communication systems and are described in detail in this section. We call them "communication patterns" as they can be seen as a kind of design patterns upon which future applications can be built.

This classification is independent of the actual communication technology and assumes only the availability of a linklayer broadcast and unicast mechanism. IEEE 802.11p [7] is a good example of a suitable communication system that is likely to be deployed in VANETs.

For each pattern we determine the following characteristics:

Purpose: Describes the overall goal of this pattern.

Communication Mechanism: Describes generic communication mechanisms and presents examples of mechanisms conforming to this pattern.

Trigger: Describes the circumstances under which the communication is typically initiated.

Direction: Communication can be either unidirectional, bidirectional with response(s) to the sender or without clear direction.

Data: Outlines typical communicated data.

Quality of Service: Describes typical capability and requirements of the communication patterns regarding metrics like message distribution success or latency.

Examples: Refers to corresponding examples of Table I.

\section{A. Beaconing}

Purpose: Continuous update of information among all neighboring nodes, e.g. to supply them with up-to-date status

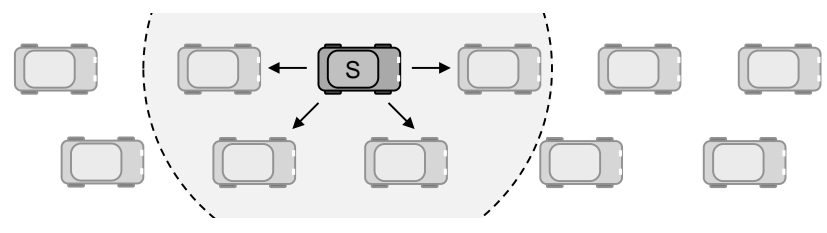

Fig. 1. Beaconing - Permanent, periodic single-hop broadcast messages

data like the position, speed, heading of a vehicle to allow for cooperative awareness.

Communication Mechanism: Data packets are sent as link layer broadcasts to all neighbors in reception range. Communication is single-hop and information in packets is typically not forwarded, even after processing by an application (Figure 1).

Trigger: Data packets are sent continuously and periodically. In few cases, the beaconing mechanism may be started by an external trigger, e.g. if an accident occurred. But in such a case, the information may just be added to the regular beacon packets.

Direction: Communication is strictly unidirectional, even if every node uses beaconing.

Data: Data in the packets is generated by the sender, usually local sensor data or derived from local sensors.

Quality of Service: Many applications using beaconing have mid-range latency requirements, e.g. in cooperative awareness applications like the highway merge assistant application, status information at neighbors need to be updated at least at the scale of seconds.

Examples: I.1, I.2.3, I.3.1-4+5-6, I.4, I.5.1-2, II.1.3, III.1, IV.1

\section{B. Geobroadcast}

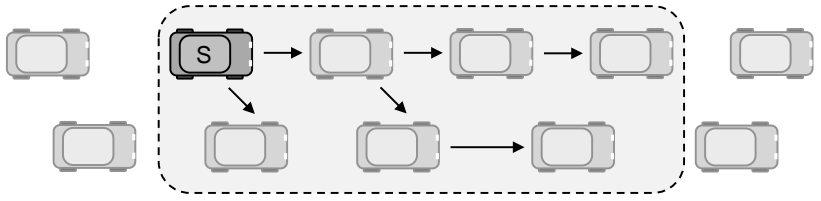

Fig. 2. Geobroadcast - Sender (S) initiated, multi-hop dissemination of a message within a geographic destination region

Purpose: Immediate distribution of information in a larger area, e.g. to inform approaching vehicles about a sudden event or abnormal road condition that needs attention by drivers.

Communication Mechanism: The sender of the message determines a destination region for the message to be sent and attaches it to the message. Then the message is sent via link layer broadcast to all immediate neighbors in transmission range. Every receiver which is located within the specified destination region forwards the unchanged message via broadcast (geographically restricted flooding, Figure 2). For a better scalability in a situation with high node density, the forwarding scheme may also be optimized to reduce redundancy, like e.g. in Gossiping [8].

Trigger: Geobroadcast messages are typically sent upon a certain external event, or in other words, Geobroadcast 
messages are not sent continuously, though messages with the same content may be repeated from time to time, e.g. in case of a work zone warning.

Direction: Geobroadcast messages are unidirectional.

Data: Messages contain data which is set by the sender. For example, in case of an accident, the crashed vehicle detects this situation using local sensors and can then send an appropriate warning message.

Quality of Service: Due to their event-based nature, Geobroadcast messages will often require very low latency of messages to inform addressed vehicles as fast as possible. On the other hand, some applications like the aforementioned work zone warning have relaxed requirements here, so messages should indicate their priority to adapt forwarding accordingly. In addition, certain applications like accident warning may need best possible delivery success to inform all addressed vehicles.

Examples: I.2.4, I.3.5, I.5, II.1, II.2.4, III.1.3

\section{Unicast Routing}

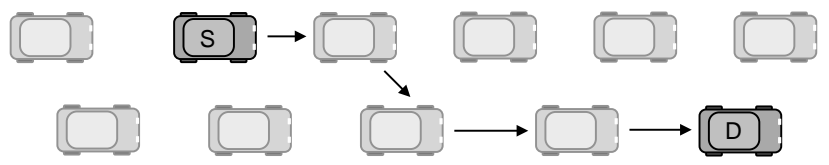

Fig. 3. Unicast routing - Communication between two endpoints S and D, single-hop or multi-hop, possibly bidirectional

Purpose: Transport of data through the ad hoc network to a certain destination (other vehicle or road side unit). The goal is to use the network for unicast transport of messages, not for distribution of messages.

Communication Mechanism: The communication may consist of only a single hop, or route messages over multiple hops towards the destination. The destination of packets is either a single node or a remote destination region (Figure 3 ). For multi-hop routing, a number of routing schemes for ad hoc networks can be considered as suitable. Position-based routing approaches have shown superior performance in contrast to topology-based approaches due to their adaptability to the high node movement dynamics in VANETs [9].

Trigger: Sending a packet via unicast routing can have various reasons, but in many cases, unicast packets will be sent upon system internal events or manual user interaction.

Direction: Transporting packets using unicast routing may be uni- or bidirectional. The latter is the case for applications that need connection-oriented communication in contrast to many warning applications for which widespread, unidirectional distribution is important.

Data: Packets carry arbitrary data, set by the sender, not altered by routing.

Quality of Service: Because many of the applications using unicast routing have no immediate relation to safety, the priority of corresponding packets is normally lower compared to safety applications. Even among unicast packets, a priority hierarchy can be determined. For example, some applications like map update can tolerate very high delays and packet retransmissions.

Examples: I.2.2+4, I.4, I.5.3, II.1.2, II.2.1-3, III.2.1+4+5, IV.

\section{Advanced Information Dissemination}

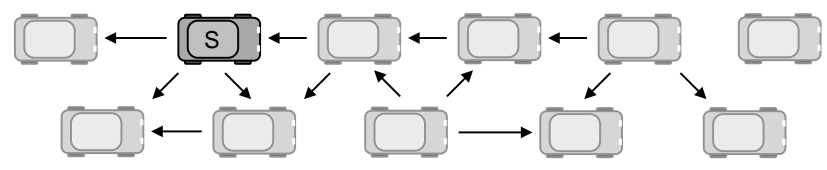

Fig. 4. Advanced message dissemination - Time-stable store-and-forward dissemination of a message, restricted by destination region or contextual relevance

Purpose: Dissemination of information among vehicles enduring a certain time, capable to bridge network partitions and prioritising information. The goal is to provide information also to vehicles which arrive later in time and which previously could not be reached due to network partitioning. In addition, the bandwidth usage should be scaled to the priority of messages in the current context of a vehicle, i.e. dissemination should send only messages with high priority at a time, if bandwidth is scarce.

Communication Mechanism: Schemes for advanced message dissemination usually use single-hop broadcasts, store messages and forward them multiple times, considering various parameters to determine when to resend a message (Figure 4). In the Abiding Geocast approach [10], messages are repeated following different strategies. For example, a message is stored at every node until a sender-defined expiry time, and repeated when a new neighbor is encountered. The approach of context-adaptive message dissemination [11] goes considerably further. The sender annotates messages with a number of meta data from its current context, like position, time, heading. All messages of all corresponding applications are lined up in a common queue and every message is rated according to its relevance in the current context. Only the most relevant message is sent at a time. The mechanism differs significantly from traditional forwarding, as the sender does not specify a destination. Instead, information is spread according to is contextual relevance and adapted to the bandwidth constraints.

Trigger: Messages are "injected" into the system based on events or discovered information. Periodic creation of messages does not make sense as the dissemination repeats messages anyway depending on their validity and relevance.

Direction: The communication is clearly unidirectional, that is, no responses or bidirectional protocols are involved.

Data: The content and original meta data of a message as created by the source is never changed. However, additional meta data to determine the relevance of a message, e.g. the last time it was sent, may be attached to a message.

Quality of Service: In context-adaptive message dissemination, priorisation is implicitly included. The mechanisms consider a widespread and time-stable dissemination of messages as more important than low latency.

Examples: I.2.1-2+4, I.5.1-2, II.1.3, III.2.3, IV.1.3 


\section{E. Information Aggregation}

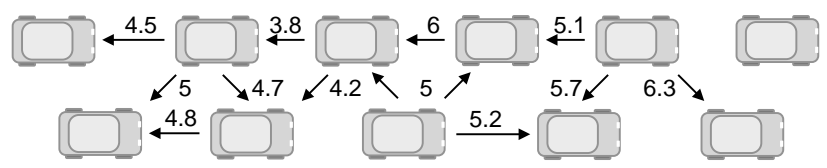

Fig. 5. Information Aggregation - abstracts from packets, concentrates on efficient distribution of information

Purpose: In contrast to all other patterns, communicated data is processed and merged by network nodes and not simply forwarded. It has a similar goal as advanced information dissemination, i.e. to spread information among vehicles. However, it can reduce communication overhead when events are detected by multiple vehicles as aggregation can also compress the data. Reduced overhead in turn decreases collision probability and dropped packets. Due to this, more bandwidth is available for dissemination of actual distinct information. For some applications, information aggregation can improve information quality, e.g. when multiple vehicles detect a single event like a traffic jam.

Communication Mechanism: The central component of information aggregation schemes is a knowledge base, which is augmented with information from local data of the own vehicle and remote data collected by other vehicles. Pieces of local knowledge are shared with others by actively sending messages regularly or upon request (Figure 5). The communication can be single or multi-hop. Messages as such are not forwarded by the receiver, instead incoming information only contributes to the local knowledge base from which new messages with merged information are created.

Trigger: Pieces of knowledge can be shared with others e.g. when a new fact like a traffic jam is detected, or exchanged periodically.

Direction: The direction of communication can not be distinguished clearly any more.

Data: The communicated data contains aggregated information, derived from multiple sources over time.

Quality of Service: In contrast to other patterns, aggregation deals with information rather than messages. Hence, information delivery, quality of information and its convergence within the network are better metrics in this case. However, this also implies that information aggregation can not be used for messages with very time-sensitive applications like emergency brake or accident warnings.

Examples: I.2.1, III.2.2-3+5

\section{CONCLUSION}

The diversity of applications envisioned for VANETs results in a challenging task for the creation of suitable communication mechanisms to enable these applications. It is particularly hard to address the different ways information is disseminated, as well as the related latency and priority requirements. Many applications do not use traditional forms of communication, but require broadcast communication and more advanced information dissemination schemes. Moreover, VANETs differ notably from other types of ad hoc networks like wireless sensor networks or mobile ad hoc networks, because of node dynamics and heterogeneity.

In this work, we classify a variety of applications into logical groups to get a more concise picture of the applications. In addition, node and network characteristics clarify influences on the design of mechanisms. Based on this analysis, we propose five "communication patterns", which satisfy the communication needs of virtually all VANET applications that are currently under discussion. These patterns can serve as a base for future development. The classification also reflects that the close coupling between applications and communication in VANETs shifts the focus to a more integrated system architecture which ultimately also includes information aggregation.

In addition, the presented communication patterns simplify the discussion on security and privacy in VANETs. The patterns can form the base for a security and privacy analysis and thus allow for a bottom-up discussion of security in order to achieve tailored security and privacy solutions. This is subject of our ongoing work e.g. in the SEVECOM $^{9}$ and PRECISA ${ }^{10}$ projects.

\section{REFERENCES}

[1] "Dedicated Short Range Communication (DSRC)," http://grouper.ieee.org/groups/scc32/dsrc/.

[2] Vehicle Safety Communications Project (VSC), "Task 3 Final Report: Identify intelligent vehicle safety applications," U.S. Department of Transportation, Tech. Rep., Mar. 2005. [Online]. Available: http://www-nrd.nhtsa.dot.gov/pdf/nrd-12/1665CAMP3web/ images/CAMP3scr.pdf

[3] Florian Doetzer, Timo Kosch, and Markus Strassberger, "Classification for traffic related inter-vehicle messaging," in Proceedings of the 5th IEEE International Conference on ITS Telecommunications, Brest, France, June 2005. [Online]. Available: http://www13.in.tum.de/personen/doetzer/publications/ Doetzer-05-ClassificationTrafficRelatedMessaging.pdf

[4] Walter Franz, Christian Wagner, Christian Maihöfer, and Hannes Hartenstein, "Fleetnet: Platform for Inter-Vehicle Communications," in Proc. 1st Intl. Workshop on Intelligent Transportation, Hamburg, Germany, Mar. 2004.

[5] Reinhold Eberhardt and Christian Maihöfer, "Virtual warning signs: A geocast enabled service for ad hoc networks," in Proceedings of the 3rd Workshop on Applications and Services in Wireless Networks (ASWN 2003), Bern, Switzerland, Jul. 2003, pp. 135-147.

[6] Jeremy J. Blum, Azim Eskandarian, and Lance J. Hoffman, "Challenges of Intervehicle Ad Hoc Networks," IEEE Transactions on Intelligent Transportation Systems, vol. 5, no. 4, pp. 347-351, Dec. 2004.

[7] IEEE 802.11p, "Wireless Access for Vehicular Environments - Draft standard."

[8] Zygmunt J. Haas, Joseph Y. Halpern, and Li Li, "Gossip-based ad hoc routing," IEEE/ACM Trans. Netw, vol. 14, no. 3, pp. 479-491, 2006. [Online]. Available: http://doi.acm.org/10.1145/1143396.1143399

[9] Walter Franz, Hannes Hartenstein, and Martin Mauve, Inter-VehicleCommunications Based on Ad Hoc Networking Principles - The FleetNet Project, W. Franz, H. Hartenstein, and M. Mauve, Eds. Universitätsverlag Karlsruhe, Nov. 2005, iSBN 3-937300-88-0. [Online]. Available: http://www.uvka.de/univerlag/frontdoor.php?source_opus=89

[10] Christian Maihöfer, Tim Leinmüller, and Elmar Schoch, "Abiding Geocast: Time-Stable Geocast for Ad Hoc Networks," in VANET '05: Proceedings of the 2nd ACM International Workshop on Vehicular Ad Hoc Networks. New York, NY, USA: ACM Press, 2005, pp. 20-29.

\footnotetext{
${ }^{9} \mathrm{http} / / / \mathrm{www}$. sevecom.org/ - Secure Vehicle Communication project

${ }^{10} \mathrm{http}: / /$ www.preciosa-project.org/ - Privacy Enabled Capability In coOperative systems and Safety Applications project
} 
[11] Timo Kosch, Christian J. Adler, Stephan Eichler, Christoph Schroth, and Markus Strassberger, "The Scalability Problem of Vehicular Ad Hoc Networks and How to Solve it," IEEE Wireless Communications, vol. 13 , no. 5, pp. 22-28, Oct. 2006. 